\title{
Elderly With Different Types of Hearing Loss and Comorbidities: Satisfaction With Hearing Aids
}

\author{
Rezvan Dashti ${ }^{1}$; Farzad Faraji Khiavi ${ }^{2, *}$; Seyyed Jalal Sameni ${ }^{3}$; Arash Bayat $^{4}$ \\ ${ }_{1}^{1}$ Musculoskeletal Rehabilitation Centre, School of Rehabilitation, Ahvaz Jundishapur University of Medical Sciences, Ahvaz, IR Iran \\ ${ }^{2}$ Department of Health Services Administration, School of Health, Ahvaz Jundishapur University of Medical Sciences, Ahvaz, IR Iran \\ 3 Department of Audiology, School of Rehabilitation, Iran University of Medical Sciences, Tehran, IR Iran \\ ${ }^{4}$ Hearing and Speech Research Center, Audiology Department, School of Rehabilitation, Ahvaz Jundishapur University of Medical Sciences, Ahvaz, IR Iran \\ ${ }^{*}$ Corresponding author: Farzad Faraji Khiavi, Department of Health Services Administration, School of Health, Ahvaz Jundishapur University of Medical Sciences, Ahvaz, IR Iran. \\ Tel: +98-6133738269, Fax: +98-6133738282, E-mail: faraji-f@ajums.ac.ir
}

Received: March 14, 2015; Revised: April 25, 2015; Accepted: May 3, 2015

\begin{abstract}
Background: Hearing loss is developing when age is rising. Initiation and progression rates of hearing loss vary among different individuals and groups.

Objectives: The current study aimed to determine satisfaction of the elderly with their hearing aids in different types of hearing loss and comorbidities.

Patients and Methods: The study was conducted on 40 elderly subjects suffering from hearing loss and using hearing aids. The data collection method included assessment of hearing loss in addition to using a questionnaire to estimate respondents' satisfaction with their hearing aids in daily life. The Persian version of the Satisfaction with Amplification in Daily Life (SADL) questionnaire was administered. The data were analyzed using descriptive and inferential statistics by SPSS software version19.

Results: The mean satisfaction scores of the elderly were $4.83 \pm 0.51$ and $5.36 \pm 0.30$ in the sensorineural loss groups. There was no significant difference between different comorbidities. There was a significant difference between satisfaction level of cost and services subscales in the symmetrical styles of hearing loss (P value $=0.04$ ).

Conclusions: The findings of the study indicated a high satisfaction of the elderly with their hearing aids, considering the type of hearing loss. Despite all the efforts to improve the audiologic services during verification process, the elderly should be consulted specifically in order to fit their hearing aid as well as their expectations from aid.
\end{abstract}

Keywords: Satisfaction; Elderly; Hearing Loss; Comorbidity

\section{Background}

According to the world health organization (WHO), three leading causes of disability around the world are hearing loss (HL), vision problems and mental disorders. Currently, 15\% of the world population, around 600 million people, has some degree of HL, and $5.4 \%$ of them have a disabling HL. Based on WHO reports, in the developed countries, only $2 \%$ of the population aged $20-24$ are suffering from hearing loss, this rate increases to $85 \%$ in the people aged $75-84(1)$.

Hearing loss can destructively affect communication. Communication is a vital skill for everyone and helps in learning and gaining knowledge and experience. When communication fails, it is difficult to establish successful business and social relationships $(2,3)$. Negative consequences of HL in adults are not confined to hearing impairment; it may also affect the individuals' socialization and performance, which can lead to depression, isolation, loss of confidence and poor performance in the elderly (3-7).

In some cases the comorbidity is the most common problem accompanying the HL, which can exacerbate the consequences of HL in individuals. Thus, it is always necessary to be aware of the kind and degree of hearing loss and other diseases in the elderly (8).

Early intervention is an integral part of aural rehabilitation programs in the elderly. Aural rehabilitation process helps people with disabilities overcome barriers to participation in daily life activities. It also helps them to resume social life. It will increase their confidence and thus improve their satisfaction level and mental health.

A number of researches focused on the outcome measurement of hearing aid fitting for many years. Several studies utilized self-assessment questionnaires either to evaluate change from pre- to post-fitting or assess aided benefits and/or satisfaction of post-fitting. Humes et al. (9) evaluated satisfaction with hearing aid in 43 elderly subjects and satisfaction with amplification in daily life (SADL) questionnaire and reported that users with various satisfaction evaluation approaches were very satisfied. Vuorialho et al. (10) assessed the effects of hearing

Copyright (C) 2015, Ahvaz Jundishapur University of Medical Sciences. This is an open-access article distributed under the terms of the Creative Commons Attribution-NonCommercial 4.0 International License (http://creativecommons.org/licenses/by-nc/4.0/) which permits copy and redistribute the material just in noncommercial usages, provided the original work is properaly cited. 
aids on hearing disability as well as quality of life and showed that using amplification decreased the number of hearing loss handicaps. Hosford-Dunn and Halpern in their research reported that SADL questionnaire can be a key standard instrument to measure satisfaction (11).

\section{Objectives}

The current study aimed to determine satisfaction of the elderly with their hearing aids in different types of hearing loss and comorbidities.

\section{Patients and Methods}

Participants consisted of 40 hearing-aid users from private hearing aids clinics volunteered for the study. The majority of the subjects were male (75\%) with the age range of 65 - 90 years.

\subsection{Procedure}

In order to measure the subjects' satisfaction with the hearing aids, the standard Persian version of SADL questionnaire was used. The questionnaire's content and face validity was approved by 5 experts, with the Cronbach's alpha $=0.8$. This instrument is designed to assess subjects' satisfaction with their hearing aids in the real life. The SADL questionnaire consists of 15 questions and provides a global score indicating overall satisfaction, as well as four separate subscale scores including positive effect, service and cost, negative features and personal image. Subjects' responses to each item were obtained using a seven-point Likert Scale ranging from "Not at all" to "Tremendously". Positive effect subscale includes six questions considering acoustical and psychological advantages. Three questions about background noise and using phone are covered in negative features subscale. The specialist's skill, hearing aid cost and repairing times are conferred in cost and services subscale and in the last subscale, personal image contains three questions about cosmetic and motivate factors and labeling while using hearing aids. The answers are equivalent to a seven-point scales, where the score with the smallest value is one, corresponds to the answer "Not at all"; the highest value is seven and corresponds to the response "Tremendously", indicating the lowest and highest satisfaction levels, respectively. Reverse scoring was considered in four questions. The SADL yields a global satisfaction score, as well as a profile of the four subscale scores. Global Satisfaction was calculated from the mean of all responded items. Subscale values were the average value of the items included in that subscale.

\subsection{Statistics}

Descriptive (mean and standard deviation) and inferential (Mann-Whitney and Kruskal-Wallis) statistics were used to analyze data by SPSS. Mann-Whitney U test was used to compare the mean scores of SADL global and subscales between mixed HL/SNHL, symmetrical/asymmetrical patterns as well as comorbidities/non-comorbidities. Kruskal-Wallis was used to compare hearing loss severity levels. The significance level was set at 0.05.

\section{Results}

Thirty-four subjects (85\%) were suffering from sensorineural hearing loss and six (15\%) from mixed hearing loss. All of the subjects were using monaural hearing aids. Thirty-eight (95\%) of them were using BTE (behind the ear) hearing aids and only two (5\%) used CIC (completely in the canal) hearing aids. The satisfaction with hearing aids in the elderly according to the type of their hearing loss is shown in Figure 1.

Considering the overall average of the scores of satisfaction with hearing aids of all the subjects based on the type of hearing loss a significant difference was observed (Figure 1). The highest level of satisfaction was found in the positive effects of using hearing aids and the lowest level of satisfaction was found in the negative features. A significant difference was observed between the satisfaction level of positive effects subscale $(P$ value $=0.03)$ and global score $(P$ value $=0.01$ )

The satisfaction with hearing aids in the elderly subjects based on audiogram pattern (symmetrical or asymmetrical) is shown in Table 1 . Twenty-four (60\%) of the subjects were suffering from symmetrical hearing loss, and 16 (40\%) from asymmetrical hearing loss. Satisfaction with hearing aids scores in the elderly in terms of services, costs, and personal image were within the same range that is, they were relatively satisfied with their hearing aids. In terms of positive effects, the level of satisfaction was high while satisfaction was in the lowest level in terms of negative features and there was a significant difference between satisfaction with the services, cost of hearing aids and hearing loss symmetry (Table 1).

Distribution of subjects' satisfaction according to the degree of hearing loss without hearing aids is shown in Figure 2.

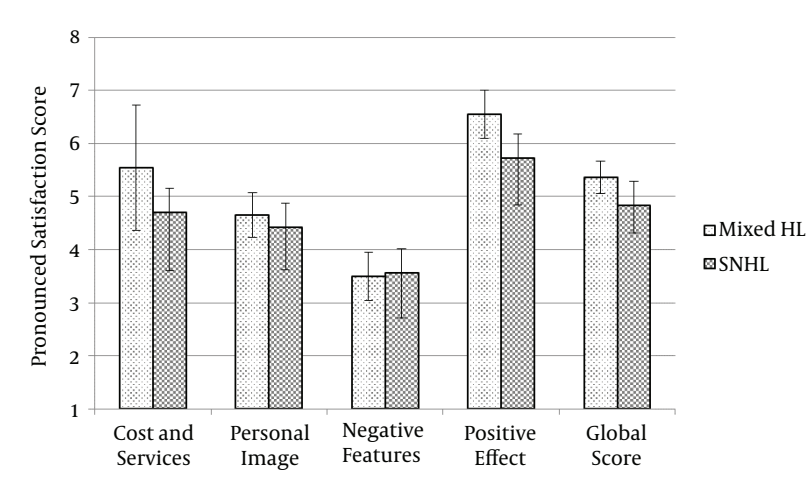

Figure 1. Satisfaction With Hearing Aids in the Elderly According to the Type of Hearing Loss 
Table 1. Satisfaction With Hearing Aids in the Elderly Based on Symmetrical or Asymmetrical Hearing Loss in Both Ears ${ }^{a, b}$

\begin{tabular}{lccc}
\hline \multirow{2}{*}{ SADL Subscale } & \multicolumn{2}{c}{ Symmetry of HL } & \multirow{2}{*}{ P Value } \\
\cline { 2 - 3 } & Symmetrical & Asymmetrical & \\
\hline Cost and services ${ }^{\mathrm{C}}$ & $5.12 \pm 1.09$ & $4.39 \pm 1.08$ & 0.04 \\
\hline Personal image & $4.50 \pm 0.66$ & $4.39 \pm 0.90$ & 0.67 \\
Negative features & $3.48 \pm 0.74$ & $3.66 \pm 0.90$ & 0.49 \\
Positive effect & $5.91 \pm 0.70$ & $5.77 \pm 1.11$ & 0.61 \\
Global score & $4.98 \pm 0.43$ & $4.80 \pm 0.63$ & 0.26 \\
\hline
\end{tabular}

a Abbreviation: HL, hearing loss; SADL, satisfaction with amplification in daily life.

$\mathrm{b}$ Values are presented as mean $\pm \mathrm{SD}$.

c $\mathrm{P}<0.05$.

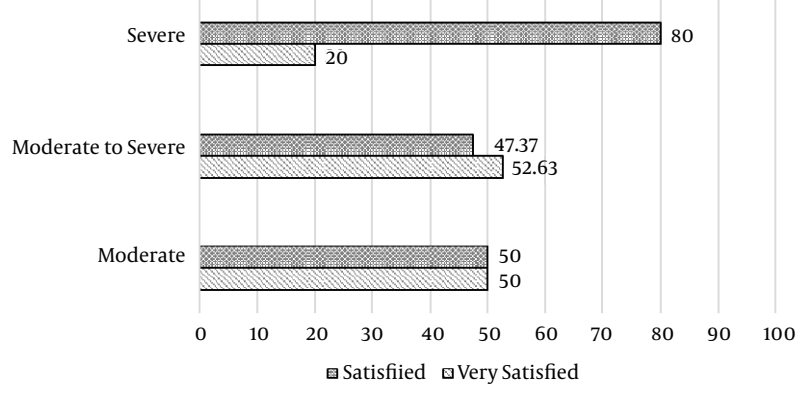

Figure 2. Percentage of Patients' Satisfaction According to the Degree of Hearing Loss Without Hearing Aids

Table 2. Satisfaction With Hearing Aids in the Elderly Considering Their Comorbidity ${ }^{\mathrm{a}}$

\begin{tabular}{lcc}
\hline SADL Subscale & With Comorbidities & No Comorbidities \\
\hline Cost and services & $5.02 \pm 1.09$ & $4.54 \pm 1.16$ \\
Personal image & $4.44 \pm 0.87$ & $4.47 \pm 0.58$ \\
Negative features & $3.59 \pm 0.83$ & $3.50 \pm 0.77$ \\
Positive effect & $5.80 \pm 0.93$ & $5.93 \pm 0.82$ \\
Global score & $4.93 \pm 0.55$ & $4.87 \pm 0.49$ \\
\hline
\end{tabular}

a Abbreviation: SADL, satisfaction with amplification in daily life.

Sixteen (40\%), nineteen (5.47\%), and five (12.5\%) subjects were suffering from moderate, moderate to severe and severe hearing loss, respectively. Eighty percent of the subjects with severe hearing loss in the study had the lowest satisfaction. The highest satisfaction rate among patients with moderate to severe hearing loss was 52.63\%. The satisfaction with hearing aids in the elderly considering their comorbidities are indicated in Table 2.

Forty percent $(n=16)$ of the elderly subjects were not suffering from comorbidity and sixty percent $(n=24)$ were suffering from diseases other than hearing loss.

In the present study, no significant differences were found between the elderly subjects' satisfaction with hearing aids and their comorbidities that is, satisfaction or dissatisfaction with hearing aids had nothing to with comorbidities or other diseases. The scores of satisfaction in terms of negative features, the user's personal image, and services and cost were in the same range which was indicating the relative satisfaction of the subjects; in terms of positive features the subjects were highly satisfied with their hearing aids (Table 2).

\section{Discussion}

The current study aimed to determine the level of satisfaction with the hearing aids among the elderly with different types of hearing loss and comorbidities. In this research, most of the studied subjects (85\%) were suffering from sensorineural hearing loss; they were less satisfied with their hearing aids in comparison with subjects suffering from mixed hearing loss. These findings are consistent with those of a study by Dell' Antonia et al. in which subjects suffering from mixed hearing loss were more satisfied with their hearing aids than the ones suffering from sensorineural (12). The main cause can be the fact that the bone conduction system in patients with mixed hearing loss is working better than the ones suffering from sensorineural hearing loss.The mean score of the subjects with severe hearing loss indicated the relative satisfaction with their hearing aids. However, compared to the overall mean score of all subjects, their scores were lower which means they were less satisfied with their aids globally. In the current study, no mean score indicated dissatisfaction with hearing aids in the elderly based on the degree of hearing loss. The obtained results were in compliance with those of the studies by Veiga et al. (13) and Carvalho (14) while in another study (12) half of the subjects suffering from severe hearing loss were satisfied with their hearing aids.

In the current research no significant differences were observed between satisfaction scores of different comorbidity groups. The subjects were in the age range of 65 90 years old and it seems that at this age they are more likely to be affected by psychological and chronic diseases of aging, thus, they respond differently to the use of hearing aids (14).

The scores of satisfaction with hearing aids in the subjects using BTE $(4.91 \pm 0.53)$ and CIC (4.80 \pm 0.28$)$ hearing aids were in the same range which showed the relative satisfaction of the subjects with the appearance of their hearing aids; this result was in compliance with those of the study by Dell' Antonia et al. (12).

The findings indicated moderate satisfaction of the elderly with their hearing aids based on the type of hearing loss. Despite all efforts to improve the rehabilitation services, in order to increase subjects' satisfaction, a specialist counseling is necessary to moderate their expectations from hearing aids.

\section{Acknowledgements}

The data used in the current paper were derived from 
MSc thesis of Rezvan Dashti, student of Ahvaz Jundishapur University of Medical Sciences.

\section{Authors' Contributions}

Rezvan Dashti: providing abstract, study concept and study design, data gathering and interpreting; Farzad Faraji Khiavi: study design, data analysis and interpretation, manuscript drafting; Arash Bayat: manuscript development and drafting; Seyyed Jalal Sameni: manuscript drafting.

\section{Funding/Support}

The current study was financially supported by Ahvaz Jundishapur University of Medical Sciences, Ahvaz, IR Iran (grant no: PHT-9305).

\section{References}

1. World Health Organization. WHO global estimates on prevalence of hearing loss. WHO: 2012.

2. Dalton DS, Cruickshanks KJ, Klein BE, Klein R, Wiley TL, Nondahl DM. The impact of hearing loss on quality of life in older adults. Gerontologist. 2003;43(5):661-8.

3. Chisolm TH, Johnson CE, Danhauer JL, Portz LJ, Abrams HB, Lesner S, et al. A systematic review of health-related quality of life and hearing aids: final report of the American Academy of Audiology Task Force On the Health-Related Quality of Life Benefits of Amplification in Adults. J Am Acad Audiol. 2007;18(2):151-83.

4. Gopinath B, Hickson L, Schneider J, McMahon CM, Burlutsky G,
Leeder SR, et al. Hearing-impaired adults are at increased risk of experiencing emotional distress and social engagement restrictions five years later. Age Ageing. 2012;41(5):618-23.

5. Gates GA, Murphy M, Rees TS, Fraher A. Screening for handicapping hearing loss in the elderly. J Fam Pract. 2003;52(1):56-62.

6. Perez E, Edmonds BA. A systematic review of studies measuring and reporting hearing aid usage in older adults since 1999: a descriptive summary of measurement tools. PLoS One. 2012;7(3):e31831.

7. Wu HY, Chin JJ, Tong HM. Screening for hearing impairment in a cohort of elderly patients attending a hospital geriatric medicine service. Singapore Med J. 2004;45(2):79-84.

8. Ozler GS, Ozler S. Quality of Social and Sexual Life in Males with Hearing Loss. Int Adv Otol. 2013;9(2):211-8.

9. Humes LE, Wilson DL, Humes AC. Examination of differences between successful and unsuccessful elderly hearing aid candidates matched for age, hearing loss and gender. Int J Audiol. 2003;42(7):432-41.

10. Vuorialho A, Karinen P, Sorri M. Effect of hearing aids on hearing disability and quality of life in the elderly. Int J Audiol. 2006;45(7):400-5.

11. Hosford-Dunn H, Halpern J. Clinical application of the SADL scale in private practice II: predictive validity of fitting variables. Satisfaction with Amplification in Daily Life. J Am Acad Audiol. 2001;12(1):15-36.

12. Dell'Antonia SF, Ikino CM, Carreirao Filho W. Degree of satisfaction of patients fitted with hearing aids at a high complexity service. Braz J Otorhinolaryngol. 2013;79(5):555-63.

13. Veiga LR, Merlo ARC, Mengue SS. Satisfaction level with hearing aid in the daily life of Army Healthcare System users. Rev Bras Ottorhinolaryngol. 2005;71(1):67-73.

14. Carvalho JSA. Satisfaction of the Elderly With Hearing Aid Provided in Tocantins State-Brazil. Int Arc Ottorhinolaryngol. 2007;11(4):416-26. 